\title{
Attenuated response to purified protein derivative in patients with rheumatoid arthritis: study in a population with a high prevalence of tuberculosis
}

\author{
D Ponce de León, E Acevedo-Vásquez, A Sánchez-Torres, M Cucho, J Alfaro, R Perich, C Pastor, \\ J Harrison, C Sánchez-Schwartz
}

Background: The purified protein derivative (PPD) skin test is the only widely used method which detects latent tuberculosis infection (LTBI) and is dependent on a normal T cell function. In rheumatoid arthritis (RA) the $T$ cell function is altered, which may result in an inability to develop an adequate PPD reaction.

Objectives: To evaluate the response to PPD in patients with RA and to compare it with that of control subjects.

Methods: 112 patients with RA and 96 healthy controls were studied. PPD $5 \mathrm{U}$ was applied using the Mantoux method, and skin reaction was measured at 72 hours. The reaction was considered negative for PPD $<5 \mathrm{~mm}$.

Results: There were no significant differences in age, sex, history of bacille Calmette-Guerin vaccination, or tuberculosis contact between the two groups. The median size of the PPD induration in the patients with RA was significantly less than that in the control group ( $4.5 \vee 11.5 \mathrm{~mm}, \mathrm{p}<0.01)$. 79 (70.6\%) patients with RA compared with $25(26 \%)$ of the control group had a negative reaction to PPD $(p<0.01)$, a response not influenced by disease activity or duration of disease in the patients with RA.

Conclusion: A PPD skin test is not an appropriate test for recognising $L T B I$ in patients with $R A$ in our population.

$\mathrm{T}$ he purified protein derivative (PPD) skin test is currently the only widely used method which detects latent tuberculosis infection (LTBI). ${ }^{1}$ A defect in the cellular immune function exists in rheumatoid arthritis (RA), ${ }^{2}$ which may result in an inability to produce an adequate response to the PPD test, even though infected by the bacteria. Since the introduction of biological treatment in patients with RA, several hundred cases of tuberculosis (TB) have been observed, mostly during the first 6 months of treatment, suggesting reactivation of LTBI. The Food and Drug Administration recommend that all patients being considered for anti-tumour necrosis factor agents should be evaluated for the risk of LTBI by a PPD skin test. ${ }^{4}$ This report evaluates the PPD reactivity in patients with RA receiving conventional drugs and compares the results with patients without RA from the same population.

\section{METHODS}

The study was conducted among 112 consecutive patients with RA and 96 immunocompetent control subjects of mestizo origin matched for age and sex, recruited from the outpatient rheumatology clinic. All participants were ethnically and socioeconomically homogeneous. Control subjects consisted of immunocompetent patients diagnosed with osteoarthritis, osteoporosis, mechanical low back pain, and tendinitis. Exclusion criteria for control subjects were the presence of diseases associated with non-specific immunosuppression (diabetes, liver or renal diseases, malnutrition, or malignant diseases) and the use of immunosuppressive drugs. The following patients were excluded: those with active TB, a known hypersensitivity to PPD, acute infections, patients in hospital, positive serology for HIV, intrahospital working personnel, and the presence of diseases associated with non-specific immunosuppression. PPD was performed using the Mantoux method ${ }^{1}$ and was measured 72 hours later. A negative PPD test was defined as $<5 \mathrm{~mm}$, in both patients with RA and control groups. A positive PPD was considered to be $\geqslant 10 \mathrm{~mm}$ in control patients and $\geqslant 5 \mathrm{~mm}$ in patients with RA. According to disease activity, patients were grouped as active or inactive. Active disease was defined as the presence of six or more swollen joints, six or more tender joints, and at least two or more of the following: morning stiffness lasting $>45$ minutes, erythrocyte sedimentation rate $>28 \mathrm{~mm} / \mathrm{lst} \mathrm{h}$, and $\mathrm{C}$ reactive protein $>50 \mathrm{mg} / \mathrm{l}$. BCG vaccination was recorded in a personal interview and by verifying the scar it makes. A $\chi^{2}$ or Fisher's exact test was used. Continuous variables were compared by Student's $t$ test. A value of $\mathrm{p}<0.05$ was considered significant.

\section{RESULTS}

According to the exclusion criteria, 28 patients were excluded from the analysis: 8 did not return for a PPD reading, 4 had other chronic medical conditions (diabetes, allergic rhinitis, asthma), 10 had malnutrition, 1 presented a hypersensitivity reaction to $\mathrm{PPD}$, and 5 had acute infections (upper respiratory and urinary tract infection). One hundred and twelve patients and 96 controls were included. There were no significant differences between patients with RA and controls in age (mean (SD) 54.7 (12.3) v 55.6 (11.8); $\mathrm{p}=0.56$ ); sex (94\% v 92\% female; $\mathrm{p}=0.58$ ); body mass index (24.6 (3.6) $v$ 24.4 (3.7); $\mathrm{p}=0.67)$; $\mathrm{BCG}$ vaccination $(91 \% v 82 \%$; $=0.14)$, or TB contact $(18 \% v 16 \% ; \mathrm{p}=0.35)$. One hundred and three (92\%) patients with RA had a positive rheumatoid factor compared with three (3\%) in the control group.

In patients with RA the mean (SD) disease duration was 15.6 (10.1) years and 33/112 (29\%) patients had active disease. Disease modifying antirheumatic drug treatment was used alone or in combination in $80 \%$, with methotrexate being the most commonly used drug $(68 \%)$. Eighty seven per cent of patients received prednisone ( $\leqslant 7.5 \mathrm{mg}$ daily).

Figure 1 shows that $79 / 112(70.6 \%)$ of patients with RA had a negative reaction to PPD $(<5 \mathrm{~mm})$ compared with 25/ $96(26 \%)$ from the control group $(\mathrm{p}<0.01)$. Seventy three (65\%) patients with RA showed no reaction to PPD (0 mm) compared with $24 / 96$ (25\%) in the control group.

Abbreviations: LTBI, latent tuberculosis infection; PPD, purified protein derivative; RA, rheumatoid arthritis; TB, tuberculosis 


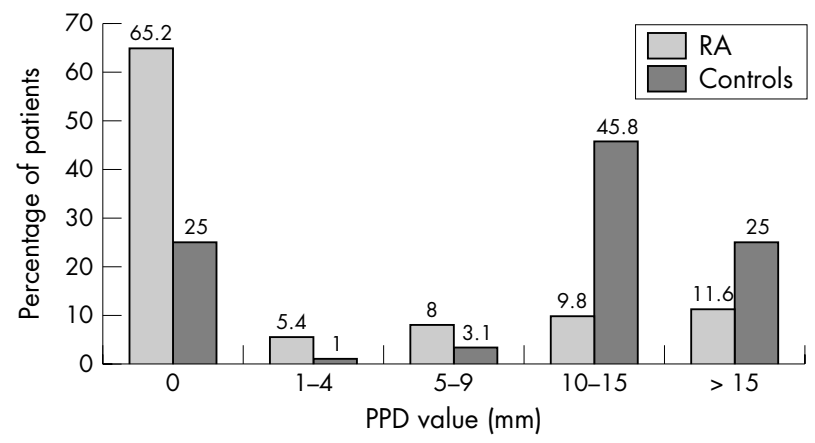

Figure 1 Induration sizes to tuberculin skin testing in patients with RA and controls.

In patients with RA a similar negative reaction to PPD $(p=0.65)$ was found in the active $(67 \%)$ and in the inactive group $(72 \%)$. To determine whether or not this difference was influenced by factors that altered the reactivity to PPD we compared the PPD positive and PPD negative groups. Although PPD negative patients with RA tended to have a longer disease duration (12.8 (9.2) v 16.7 (10.3) years), the difference was not significant $(\mathrm{p}=0.06)$. There were no differences in age $(54.4$ (12.4) $v 54.8$ (12.3) years; $\mathrm{p}=0.87)$; albumin $(40$ (3) $v 39$ (4) g/ $\mathrm{l} ; \mathrm{p}=0.87)$; active disease $(33 \% \vee 28 \% ; \mathrm{p}=0.65)$; immunosuppressive treatment $(81 \% \vee 79 \% ; \mathrm{p}=0.99)$; use of prednisone $(85 \% v 89 \% ; \mathrm{p}=0.25)$, and history of BCG vaccination $(89 \% v$ $96 \% ; \mathrm{p}=0.36$ ) between the two groups. When the group who did and did not receive prednisone were compared, 74/98 (75\%) of those using the drug had a negative reaction to PPD, whereas $9 / 14(64 \%)$ in the group not receiving prednisone had a negative reaction to $\mathrm{PPD}$.

\section{DISCUSSION}

The Hospital Nacional Guillermo Almenara Irigoyen, a 900 bed referral hospital, belongs to the social security system, serving 7 million people or one third of the Peruvian population. The incidence of TB in patients with RA in our hospital was 216/ 100000 (Acevedo-Vasquez et al, unpublished), whereas in the general population it was $133.6 / 100000 .^{5}$

Subjects with a negative reaction to PPD are those who are not infected or unable to develop a delayed hypersensitive skin reaction. ${ }^{6}$ In this prospective case-control study we found a high negative reaction to PPD in patients with RA.

More than $90 \%$ of the patients taking part in the study had a history of BCG vaccination, which as a rule leads to a positive PPD skin test. ${ }^{7}$ However, as demonstrated by Wang et al, ${ }^{8}$ when the PPD test is taken 15 years or more after vaccination, the vaccination does not have any influence on PPD reactivity. In Peru vaccination, in general, is performed shortly after birth.

The prevalence of LTBI in the general population in our country is $68 \%$ in people over 25 years of age, with a BCG vaccination cover of $87 \%$ in the general population ${ }^{9}$ and $74 \%$ in health workers from our hospital (Acevedo-Vasquez et al, unpublished). In our group of control patients we found a prevalence of positive PPD of $71 \%$ compared with only $29 \%$ in the RA group. This difference is in contrast with that reported by Wolfe et al, ${ }^{10}$ who found no decrease in PPD positivity between patients with RA (9.2\%) and background rates in the American population. This disparity with our results probably is due to the much higher TB prevalence in our group and the way in which the information was obtained in the other group, mainly retrospectively and by telephone contact.

It has been reported that patients receiving daily corticosteroids at a dose $<15 \mathrm{mg} /$ day will not suppress cutaneous delayed hypersensitivity, including tuberculin reactivity. ${ }^{11}$ All of our patients received prednisone at doses of $\leqslant 7.5 \mathrm{mg} /$ day.
Epidemiologically, it is essential to identify those who have LTBI, because treating this group of patients reduces the progression of active TB by between 50 and $80 \%$, and its effect probably persists for a lifetime. ${ }^{1}$

TB is endemic in Peru, and we found a high LTBI rate in the control group. We should have found a similar rate in the patients with RA. We demonstrated a markedly lower incidence among patients with RA (30\%) than among controls $(70 \%)$ that was unrelated to treatment or disease activity. Thus, the possibility that PPD testing may be misleading should be borne in mind for patients who have RA. Although there are in vitro diagnostic methods-not yet validated-like lymphoproliferation tests to TB-specific antigens, ${ }^{12}$ devised to permit better diagnosis of LTBI, their use is hindered by their high cost.

\section{CONCLUSIONS}

We consider that it is not appropriate to use PPD to recognise LTBI in patients with RA in our population. However, until other more sensitive diagnostic tests are available for the identification of LTBI, the PPD standard test should continue to be used.

\section{ACKNOWLEDGEMENTS}

Supported by a grant from the Social Security System, EsSalud-Peru, Universidad Nacional Mayor de San Marcos.

We are indebted to Dr Frank A Wollheim, who reviewed the manuscript and provided many useful suggestions.

\section{Authors' affiliations}

D Ponce de León, E Acevedo-Vásquez, A Sánchez-Torres, M Cucho,

J Alfaro, R Perich, C Pastor, J Harrison, C Sánchez-Schwartz, Division of Rheumatology, Hospital Nacional Guillermo Almenara Irigoyen,

EsSalud, Lima; Universidad Mayor Nacional de San Marcos, Lima, Peru

Presented in part at the 67th Annual Scientific Meeting of the American College of Rheumatology, Orlando, Fl, October, 2003.

Correspondence to: Professor E Acevedo-Vásquez, Academic Department of Rheumatology, Hospital Nacional, Guillermo Almenara Irigoyen, Avenida Grau 800, La Victoria, Lima, Peru; edacvas@terra.com.pe

Accepted 25 January 2005

\section{REFERENCES}

1 American Thoracic Society. Targeted tuberculin testing and treatment of latent tuberculosis infection. AmJRespCritCareMed 2000;161:221-47.

2 Panayi G, Corrigall V, Pitzalis C. Pathogenesis of rheumatoid arthritis. The role of T cells and others beasts. Rheum Dis Clin North Am 2001;27:1-18.

3 Keane J. Tuberculosis associated with infliximab. N Engl J Med 2001;345:1098-104.

4 Cush JJ, Matteson EL. Hotline 2001 FDA Advisory Committee Reviews Safety of TNF Inhibitors. http://www.rheumatology.org/Publications/hotline/ 0901 inf.asp, accessed 18 July 2005..

5 Alarcon E. Evaluacion epidemiologica y operacional del Programa Nacional de Control de Tuberculosis en el Peru al Año 2000, Informe Anual 2000 Programa Nacional de Control de Enfermedades Transmisibles 2001:25-39.

6 Yates A, DeShazo R. Delayed hypersensitivity skin testing. Immun Allergy Clinics North America $2001 ; 21: 1-12$.

7 APIC Guidelines Committee. APIC position paper: Responsibility for interpretation of the PPD tuberculin skin test. Am J Infect Control 1999;27:56-8.

8 Wang L, Turner M, Elwood R. A meta-analysis of the effect of Bacille Calmette Guerin vaccination on tuberculin skin test measurements. Thorax. 2002;57: 804-9; erratum, 2003;58:188.

9 Getchell WS, Davis CE, Gilman J. Basic epidemiology of tuberculosis in Peru: a prevalence study of tuberculin sensitivity in a Pueblo joven. Am J Trop Med Hyg 1992;47:721-9.

10 Wolfe F, Michaud K, Anderson J, Urbansky K. Tuberculosis infection in patients with rheumatoid arthritis and the effect of infliximab therapy. Arthritis Rheum 2004;50:372-9.

11 Schatz P, Patterson R, Kloner R, Falk J. The prevalence of tuberculosis and positive tuberculin skin test in a steroid-treated asthmatic population. Ann Intern Med 1976;84:261-5.

12 Mori T, Sakatani M, Yamagishi F, Takashima T. Specific detection of tuberculosis infection. An interferon- $\gamma$-based assay using new antigens. Am J Respir Crit Care Med 2004;170:59-64. 